Bangladesh J. Plant Taxon. 19(2): 119-122, 2012 (December)

(C) 2012 Bangladesh Association of Plant Taxonomists

\title{
NINETEEN NEW COMBINATIONS AND A NEW NAME IN BREYNIA J.R. FORST. \& G. FORST. (PHYLLANTHACEAE) FROM INDIAN SUBCONTINENT
}

\author{
T. Chakrabarty ${ }^{1}$ AND N.P. BALAKRISHNAN \\ Botanical Survey of India, Industrial Section, Indian Museum, 1 Sudder Street, \\ Kolkata 700 016, India
}

Keywords: Phyllanthaceae; Breynia; New combinations; New name; Sauropus.

\begin{abstract}
Nineteen new combinations and one new name are proposed in Breynia for taxa previously treated in Sauropus.
\end{abstract}

\section{Introduction}

The concept of the family Euphorbiaceae has changed as a result of evidence obtained from molecular studies and the current trend is to divide the family into several segregate families and merger of several genera. Consequent upon the creation of the family Phyllanthaceae based on the subfamily Phyllanthoideae of Euphorbiaceae, the genera Breynia, Glochidion and Sauropus were combined with Phyllanthus (Hoffmann et al., 2006; Kathriarachchi et al., 2006). However, latest molecular studies by Pruesapan et al. (2008) indicated the need for a relook into these mergers. Pruesapan (2010) has expressed reservations on the advisability of these mergers and made some logical suggestions. It is necessary to quote the statement of Pruesapan (2010) as follows:

"Molecular phylogenetic studies focused on the genus Phyllanthus L. (Phyllanthaceae) showed that Sauropus Blume (including Synostemon F.Muell.) and its related genera Breynia J.R. Forst. \& G. Forst. and Glochidion J.R. Forst. \& G. Forst. should be united with Phyllanthus to create a monophyletic genus. The relationships within Sauropus and its relationship with Breynia were studied to test/corroborate such a broad definition of Phyllanthus. The molecular phylogenetic analyses show that Sauropus in the broad sense is composed of two distinct groups, the former Australian Synostemon and the Southeast Asian Sauropus in the strict sense with the monophyletic Breynia embedded in the latter. As the phylogeny of the species rich Phyllanthus is still far from completed and the results here strongly support the distinction of monophyletic groups such as Glochidion, former Synostemon, and Sauropus/Breynia. These genera are recognizable, while union with Phyllanthus (suggested by Hoffmann and co-authors in 2006) will turn the latter into an unrecognizable monolithic giant of a genus. It is a much better strategy to use the complete phylogeny of Phyllanthus to render it into smaller, monophyletic genera that can be characterized. The present study shows Synostemon has to be recognised again on generic level. Breynia, the older name, is nested within Sauropus, leading us to transfer the latter to Breynia. Within this broadened Breynia, two subgenera and two sections are distinguished, subgenus Sauropus and subgenus Breynia with section Cryptogynium and section Breynia under the latter”.

${ }^{1}$ Corresponding author. Email: tchakrab@gmail.com 
A revision of the family Phyllanthaceae for Indian subcontinent is nearing completion and the authors favour and accept the concept of Pruesapan (2010) which necessitates the transfer of the species of Sauropus of Indian subcontinent to Breynia. The necessary new combinations and a new name proposed here are given below.

\section{New combinations}

1. Breynia androgyna (L.) Chakrab. \& N.P. Balakr., comb. nov.

Clutia androgyna L., Mant. Pl. 1: 128. 1767.

Type: "Habitat in India”(van Welzen, Blumea 48: 340. 2003), Herb. Linn. No. 1206.14 (LINN lecto).

Sauropus androgynus (L.) Merr., Bull. Bur. Forest. Philipp. Islands 1: 30. 1903.

2. Breynia assimilis (Thwaites) Chakrab. \& N.P. Balakr., comb. nov.

Sauropus assimilis Thwaites, Enum. Pl. Zeyl. 4: 284. 1861. Type: Sri Lanka, 1857, Thwaites CP 2855 (CAL syn, G-DC microfiche! syn, K 000246336 photo! syn, P 00318208 syn).

3. Breynia bacciformis (L.) Chakrab. \& N.P. Balakr., comb. nov.

Phyllanthus bacciformis L., Mant. Pl. 2: 294. 1771. Type: "Habitat in Tranquebaria" (Scott in Bosser et al., Fl. Mascareignes 160: 37. 1982), König s.n., Herb. Linn. No. 1105.6 (LINN lecto).

Sauropus bacciformis (L.) Airy Shaw, Kew Bull. 35: 685. 1980 \& 36: 343. 1981.

Agyneia bacciformis (L.) Blume, Bijdr. Fl. Ned. Ind. 595. 1826.

Synostemon bacciformis (L.) G.L.Webster, Taxon 9: 26. 1960, in adnot.

4. Breynia bicolor (Craib) Chakrab. \& N.P. Balakr., comb. nov.

Sauropus bicolor Craib, Bull. Misc. Inform. Kew 1914: 11. 1914. Type: Thailand, Chiengmai, Doi Suthep, Kerr 651 (K lecto; BM, K, A 00135515 isolectotypes).

5. Breynia bishnupadae (M. Gangop. \& Chakrab.) Chakrab. \& N.P. Balakr., comb. nov.

Sauropus bishnupadae M. Gangop. \& Chakrab. apud Chakrab. \& M. Gangop., J. Econ. Taxon. Bot. 20: 524, 544, f. 2 A-D. 1996. Type: India, Sikkim, Gulma, n.d., Modder 114 (CAL holo).

6. Breynia brevipes (Müll.-Arg.) Chakrab. \& N.P. Balakr., comb. nov.

Sauropus brevipes Müll.-Arg., Linnaea 32: 73. 1863. Type: Myanmar, Prome, 16 Sept. 1826, Wallich 7918 B (G-DC microfiche! holo, K-WALL microfiche! iso).

7. Breynia compressa (Müll.-Arg.) Chakrab. \& N.P. Balakr., comb. nov.

Sauropus compressus Müll.-Arg. in DC., Prodr. 15(2): 243. 1866. Type: India, Sikkim, 1861, J.D.

Hooker s.n. (Sauropus no. 8) (K 000246346-7 photo! syn, G-DC microfiche! syn, P 00237393-4 syn); West Bengal, Darjeeling dist., Pankhabari, J.D. Hooker s.n. (K 000246345 photo! syn).

S. quadrangularis (Willd.) Müll.-Arg. var. compressus (Müll.-Arg.) Airy Shaw, Kew Bull. 26: 337. 1972.

8. Breynia compressa (Müll.-Arg.) Chakrab. \& N.P. Balakr. var. puberula (Kurz) Chakrab. \& N.P. Balakr., comb. nov.

Sauropus quadrangularis (Willd.) Müll.-Arg. var. puberulus Kurz, Forest Fl. Burma 2: 350. 1877;

Type: Myanmar, Pegu Yomah, 25 Jan. 1869, Kurz 1586 (CAL holo).

S. compressus var. puberulus (Kurz) Chakrab. \& M. Gangop., J. Econ. Taxon. Bot. 20: 528. 1996.

9. Breynia garrettii (Craib) Chakrab. \& N.P. Balakr., comb. nov.

Sauropus garrettii Craib, Bull. Misc. Inform. Kew 1914: 284. 1914. Type: Thailand, Doi Angka, Me Wang drainage, 7 Sept. 1910, Garrett 37 (K holo, BM 000606479 iso, CAL iso, L iso). 
10. Breynia gour-maitii (Chakrab. \& M. Gangop.) Chakrab. \& N.P. Balakr., comb. nov.

Sauropus gour-maitii Chakrab. \& M. Gangop., J. Econ. Taxon. Bot. 20: 529, f. 5 A-E. 1996. Type : India, Kerala, Trivandrum dist., Way to Chamunji, 19 May 1979, Mohanan 61883 (CAL holo; $\mathrm{MH}$ iso).

11. Breynia macrantha (Hassk.) Chakrab. \& N.P. Balakr., comb. nov. Sauropus macranthus Hassk., Retzia 1: 166. 1855. Type: Indonesia, Hortus Bogorensis, Teysmann s.n. (L, n.v. iso).

12. Breynia po-khantii (Chakrab. \& M. Gangop.) Chakrab. \& N.P. Balakr., comb. nov.

Sauropus po-khantii Chakrab. \& M. Gangop., J. Econ. Taxon. Bot. 20: 531, f. 7. 1996. Type: Myanmar, Tenasserim, Mergui dist., Chaegleya, 6 May 1932, Maung Po Khant 13451 (CAL holo).

13. Breynia quadrangularis (Klein ex Willd.) Chakrab. \& N.P. Balakr., comb. nov.

Phyllanthus quadrangularis Klein ex Willd., Sp. Pl. ed. 4, 4: 585. 1805. Type: India Orientali, Klein s.n. (B-WILLD, herb. cat. no. 17985 microfiche! holo).

Sauropus quadrangularis (Klein. ex Willd.) Müll.-Arg., Linnaea 32: 73.1863 \& in DC., Prodr. 15(2): 242. 1866.

14. Breynia repanda (Müll.-Arg.) Chakrab. \& N.P.Balakr., comb. nov.

Sauropus repandus Müll.-Arg., Flora 55: 2. 1872. Type: India, Sikkim, near Nohore, Pashok, 2400 - 4000 ft., T. Anderson 922 (B syn, CAL - right hand side specimen, syn).

15. Breynia retroversa (Wight) Chakrab. \& N.P. Balakr., comb. nov.

Sauropus retroversus Wight, Icon. Pl. Ind. Orient. 6: 6, t. 1951(left). 1853. Type: Sri Lanka, Walker 2754 (K 000246332 photo! syn); ibid., Thwaites CP 3134 (CAL syn, G-DC microfiche! syn, K 000246330 photo! syn, P 00318236-7 syn).

16. Breynia rigida (Thwaites) Chakrab. \& N.P. Balakr., comb. nov.

Sauropus rigidus Thwaites, Enum. Pl. Zeyl. 4: 284. 1861. Type: Sri Lanka, 1856, Thwaites CP 2135 (K 000246350 syn; CAL -2 sheets syn, BM syn, G-DC microfiche! syn, P 0023739897-8 syn).

17. Breynia saksenana (Manilal et al.) Chakrab. \& N.P. Balakr., comb. nov.

Sauropus saksenanus Manilal et al., J. Indian Bot. Soc. 64: 294. 1985 (as saksenianus). Type: India, Kerala, Silent Valley, Nilikkal, 1150 m, 6 Aug. 1982, S. V. Prasannakumar 10398 (CAL holo, CALI iso).

18. Breynia stipitata (Hook.f.) Chakrab. \& N.P. Balakr., comb. nov.

Sauropus stipitatus Hook. f., Fl. Brit. India 5: 333. 1887. Type: India, Darjeeling, Griffith KD 4827 (CAL iso, K 000246337 photo! holo).

19. Breynia trinervia (Hook. f. \& Thomson ex Müll.-Arg.) Chakrab. \& N.P. Balakr., comb. nov. Sauropus trinervius Hook. f. \& Thomson ex Müll.-Arg., Linnaea 32: 72.1863 (as trinervis). Type: India, Khasi Hills, Hooker \& Thomson s.n. (CAL syn, G-DC microfiche! syn, K syn). HBC, Wallich 7922 A (K 000246342-3 syn). Bangladesh, Silhet, 1835, Wallich 7922 B (CAL syn, GDC microfiche! syn, K 000246341-44 photo! syn). 


\section{New name}

Breynia macrocalyx Chakrab. \& N.P. Balakr., nom. nov.

Sauropus rhamnoides Blume, Bijdr. Fl. Ned. Ind. 596. 1826, non Breynia rhamnoides (Willd.)

Müll.-Arg. 1866. Type: Java, Montis Salak, Blume s.n. (L lecto n.v., P 00360994 isolecto).

\section{References}

Hoffmann, P., Kathriarachchi, H. and Wurdack, K.J. 2006. A phylogenetic classification of Phyllanthaceae (Malpighiales; Euphorbiaceae sensu lato). Kew Bull. 61: 37-53.

Kathriarachchi, H., Samuel, R., Hoffmann, P., Mlinarec, J., Wurdack, K.J., Ralimanana, H., Stuessy, T.F.. and Chase, M.W. 2006. Phylogenetics of tribe Phyllantheae (Phyllanthaceae; Euphorbiaceae sensu lato) based on nrITS and plastid matK DNA sequence data. Amer. J. Bot. 93(4): 637-655.

Pruesapan, K. 2010. The rise and fall of Sauropus (Phyllanthaceae): a molecular phylogenetic analysis of Sauropus and allies. Doctoral thesis: Netherlands Centre for Biodiversity Naturalis (section NHN), PITA group, Leiden University Branch (Unpublished).

Pruesapan, K., Telford I.R.H., Bruhl, J.J., Draisma, S.G.A. and van Welzen, P.C. 2008. Delimitation of Sauropus (Phyllanthaceae) based on plastid matK and nuclear ribosomal ITS DNA sequence data. Ann. Bot. 102(6): 1007-1008.

(Manuscript received on 23 January 2011; revised on 31 October 2012) 